\title{
Analysis of current indications to bilateral adrenalectomy
}

\section{Maria Kurowska, Joanna Malicka, Agnieszka Zwolak, Jerzy S. Tarach Department of Endocrinology, Medical University, Lublin, Poland}

Introduction. Bilateral adrenalectomy [BA] is rarely applied as a therapeutic procedure. It serves as a life-saving treatment in chosen patients with persistent Cushing's disease after an ineffective pituitary surgery or in patients with ectopic ACTH production. Other indications for BA are: bilateral adrenocortical adenomas, congenital adrenal hyperplasia and bilateral pheochromocytoma in patients with hereditary paragangliomapheochromocytoma [PPS/PGL] syndromes. It is also a procedure of the first choice in bilateral metastatic adrenal neoplasms.

The aim of the study was to determine the indications to BA in patients hospitalized in our ward between 2001-2012.

Material and methods. 12 patients [7F; 5M] aged 19-72 years. Studies were based on retrospective evaluation and analysis of medical records.

Group 1 covered 7 patients [4F; 3M] with bilateral pheochromocytoma [in the course of MEN2 syndrome - 4 patients, PPS/PGL- one patient and von Hippel Linadu - one patient and one woman with sporadic form of bilateral pheochromocytoma].

\section{Tab.1 Patients with bilateral pheochomocytomas}

\begin{tabular}{|l|c|c|c|c|}
\hline n. & patient & gender & age & \multicolumn{1}{c|}{ diagnosis } \\
\hline 1. & KM & male & 32 & PPS/PGL syndrome \\
\hline 2. & KM & male & 19 & Von Hippel-Lindau syndrome \\
\hline 3. & KE & female & 29 & MEN 2 A \\
\hline 4. & PM & female & 26 & MEN 2A \\
\hline 5. & PZ & male & 24 & MEN 2A \\
\hline 6. & PW & male & 54 & MEN 2A \\
\hline 7. & Ł.Z. & female & 54 & Bilateral pheochormocytoma \\
\hline
\end{tabular}

In group 2 the indications to BA were adrenal metastases of renal clear cell carcinoma in two cases and a metastasis to one adrenal gland coexisting with adenoma of the other adrenal gland in one case.

Tab.2 Patients with bilateral adrenal metastases

\begin{tabular}{|c|c|c|c|c|}
\hline n. & patient & gender & age & diagnosis \\
\hline 1. & $J B$ & female & 64 & Carcinoma clarocellulare \\
\hline 2. & WL & male & 72 & Kidney cancer \\
\hline 3. & MK & Male & 45 & Carcinoma clarocellulare \\
\hline
\end{tabular}

Conclusions. Currently, the prevalent indication to BA is bilateral pheochromocytoma, mostly in the course of underlying hereditary neoplasmatic syndrome.
Group 3 covered 2 women with Cushing's disease. One of them was operated 31 years earlier propter corticotroph microadenoma and the second - because of atypical pituitary macroadenoma refractory to radiation, neurosurgical and pharmacological treatment.

Tab.3 Patients with corticotroph adenoma (Cushing's disease)

\begin{tabular}{|c|c|c|c|c|}
\hline n. & patient & gender & age & diagnosis \\
\hline 1. & FU & female & 53 & Corticotroph microadenoma \\
\hline 2. & MB & female & 58 & Corticotroph macroadenoma \\
\hline
\end{tabular}

Ryc.1 Frequency of particular indications to BA in studied group of patients

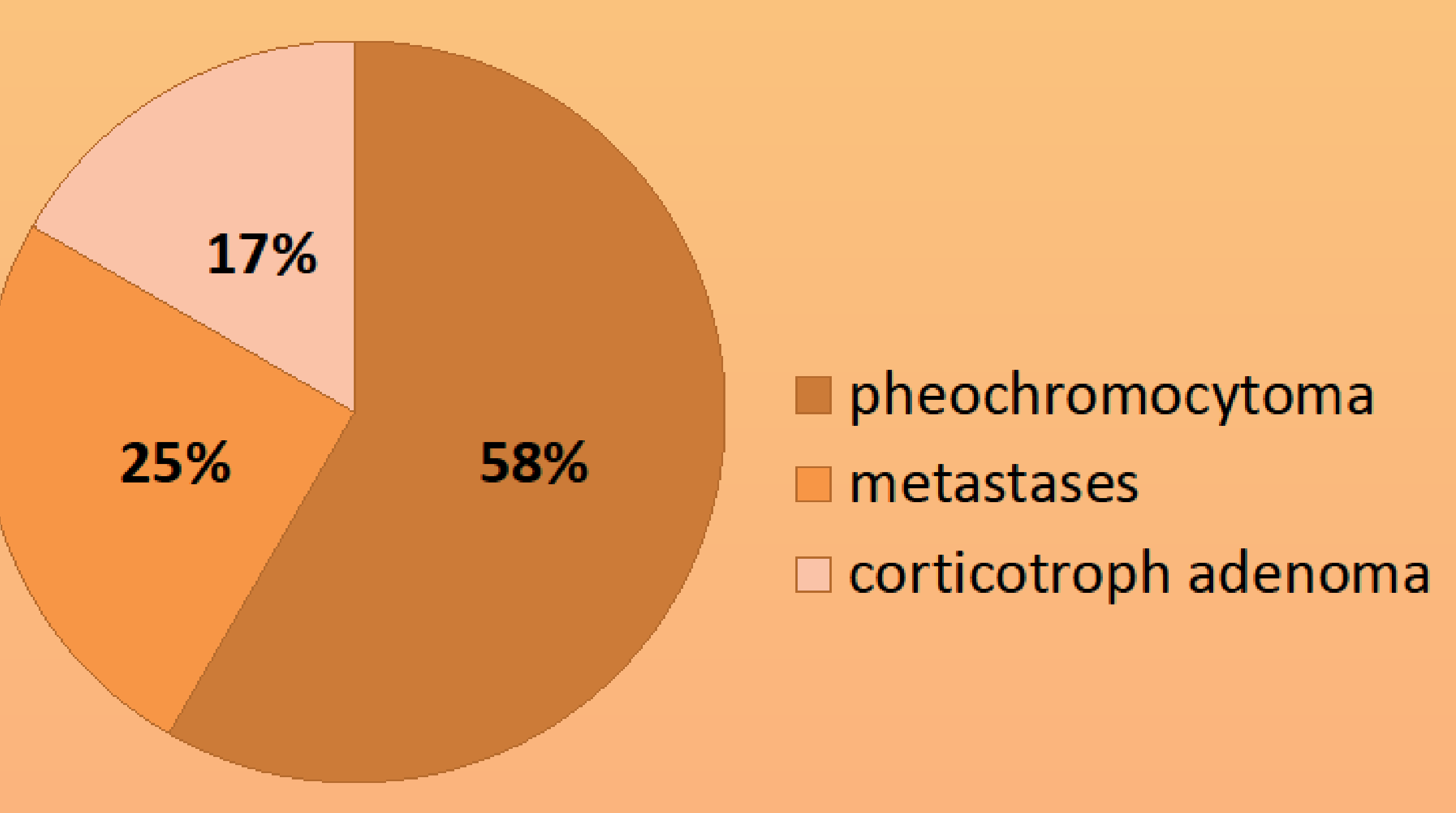

Ryc.2 Frequency of hereditary and acquired disorders as indications to BA

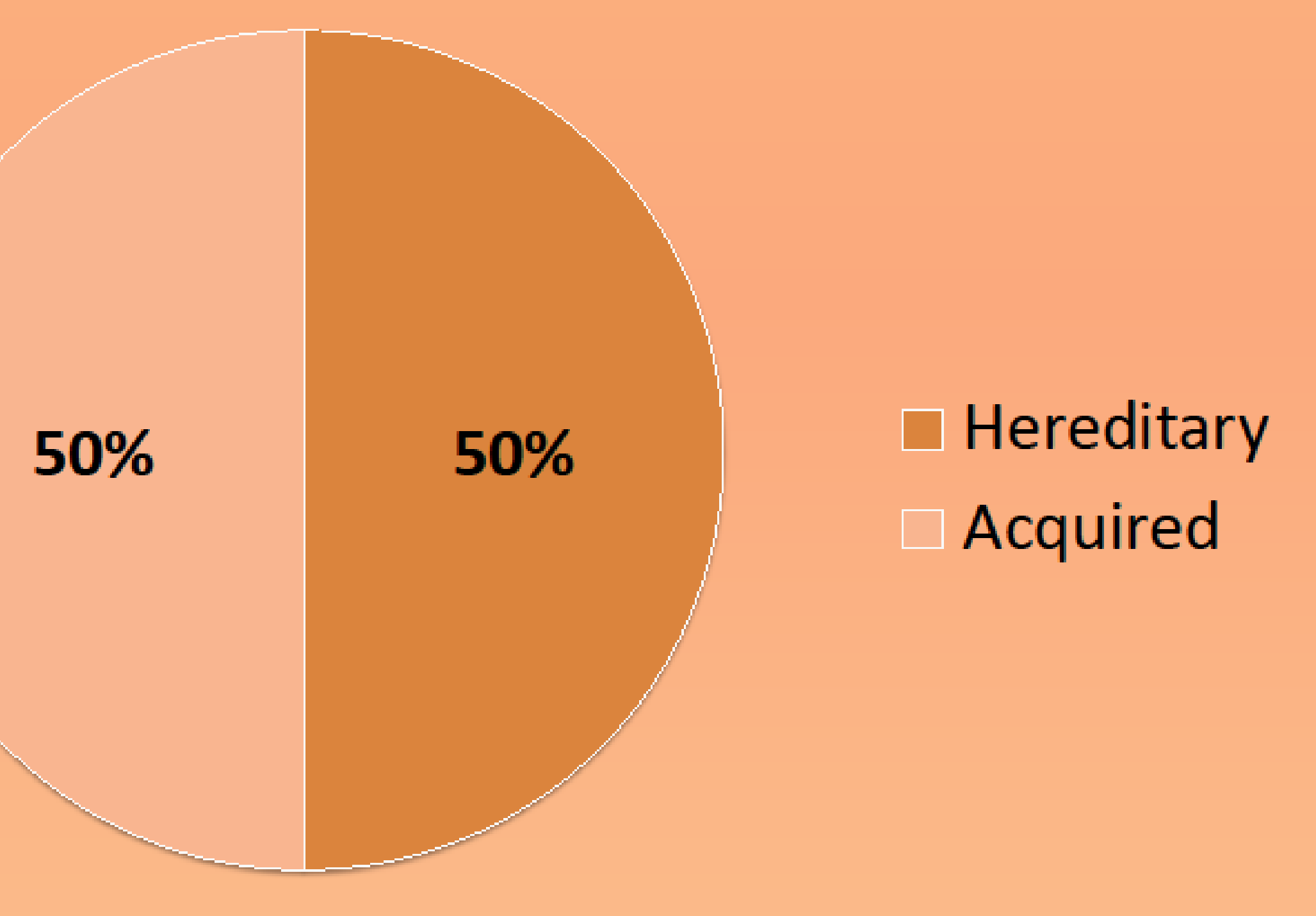

Ryc.3 Prevalence of particular acquired causes of BA

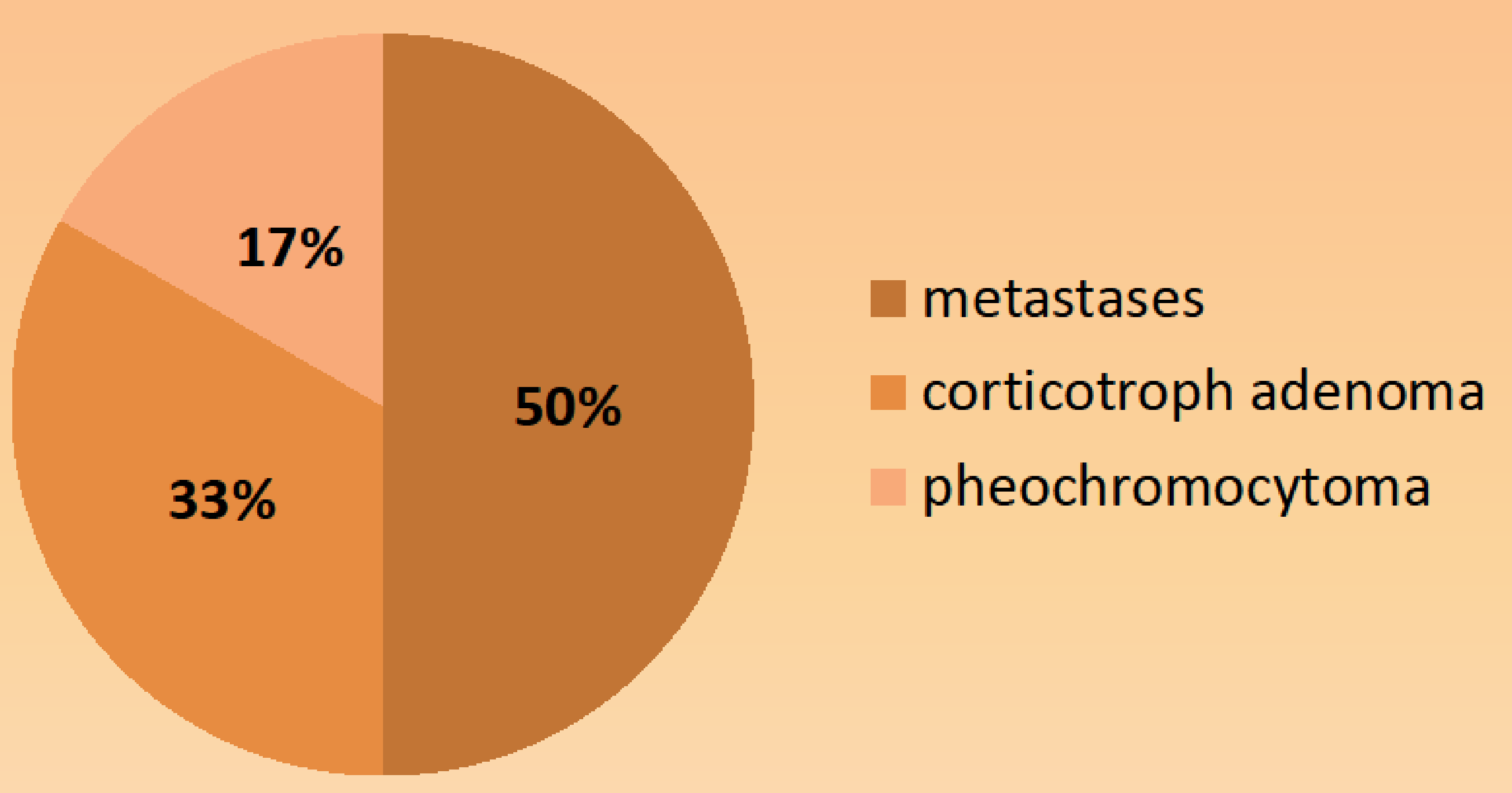

Patients with congenital disorders, Cushing's disease and one with metastasis and adenoma, underwent synchronous bilateral or two-stage adrenal surgery. Female with sporadic bilateral pheochromocytoma was operated twice at interval of 10 years and 2 patients with adrenal metastases at intervals of 3-4 years. Two patients [ $\mathrm{M}$ with renal carcinoma and $\mathrm{F}$ with atypical pituitary adenoma] died respectively after one and 3 years due to the progression of the underlying disease. The remaining patients are still alive [from 2 to 31 years after a surgery]. 